\title{
$\mathrm{Cu}$ 催化的单电子转移活性自由基聚合制备石墨烯/聚 $N, N$-二甲基 丙烯酰胺复合体系
}

\author{
王松昒郭冠南杨东胡建华* \\ (聚合物分子工程国家重点实验室 复旦大学高分子科学系上海 200433)
}

\begin{abstract}
摘要 以天然石墨为起始原料, 采用改进的 Hummer 方法, 通过强酸氧化水解和超声分散制备了氧化石墨烯, 然后通 过肼还原和重氮化反应得到含有羟基的石墨烯 $(\mathrm{G}-\mathrm{OH})$ ，再通过酯化反应在石墨烯表面上引入了 $\alpha$-氯代羰基，从而得到 了含有单电子转移活性自由基聚合(SET-LRP)引发基团的石墨烯(G-Cl). 最后，在石墨烯表面原位引发 $N, N$-二甲基丙烯 酰胺(DMAA)单体的 SET-LRP 聚合, 得到了 G-PDMAA 复合材料, G-PDMAA 在常规的有机溶剂和水中具有良好的分 散性.
\end{abstract}

关键词 石墨烯; 氧化石墨烯; $N, N$-二甲基丙烯酰胺; 单电子转移活性自由基聚合(SET-LRP)

\section{Preparation of Graphene/Poly(N,N-dimethylacrylamide) Nanocomposite via Cu-Catalyzed Single-Electron Transfer Living Radical Polymerization}

\author{
Wang, Songyang Guo, Guannan Yang, Dong Hu, Jianhua* \\ (State Key Laboratory of Molecular Engineering of Polymers, Department of Macromolecular Science, \\ Fudan University, Shanghai 200433)
}

\begin{abstract}
Exfoliated graphene oxide (GO) sheets were prepared from natural graphite by a modified Hummer's method, which included oxidative hydrolysis under strong acidic conditions and ultrasonic dispersing. GO was firstly reduced by hydrazine and then followed by a diazonium addition reaction to give graphene containing hydroxyls (G-OH), which was converted to graphene with singel eclectron transfer-living radical polymerization (SET-LRP) initiating groups via esterification with 2-chloropropionyl chloride. Finally, poly( $N, N$-dimethylacrylamide) (PDMAA) chains were grown from the surface of graphene via in situ SET-LRP to obtain graphene/PDMAA (G-PDMAA) nanocomposite. G-PDMAA nanocomposite showed good dispersity in common organic solvents and aqueous solution.
\end{abstract}

Keywords graphene; graphene oxide; $N, N$-dimethylacrylamide; singel eclectron transfer-living radical polymerization (SET-LRP)

碳元素 $(\mathrm{C})$ 是自然界最为普遍也最奇妙的元素, 不 仅可以构成已知世界上最硬的金刚石，也可以形成较软 的石墨材料. 在近二十年来, 多种新型碳基材料的发现, 如 1985 年美国科学家发现的富勒烯 ${ }^{[1]}$ 和 1991 年日本 科学家发现的碳纳米管 ${ }^{[2]}$, 使碳纳米材料一直处于科学 界的前沿领域并且在世界范围内引起持续的研究热 潮 $^{[3 \sim 7]} .2004$ 年, Geim 和 Novoselov 等 ${ }^{[8]}$ 通过机械剥离石 墨，得到了单层的石墨烯(graphene)，作为碳材料家族 的一名新成员, 它的发现, 不仅使碳材料家族更加丰富, 构成了从零维的富勒烯、一维的碳纳米管、二维的石墨 烯到三维石墨的完整体系，而且其独有的纳米结构及出 色的力学、热学、电学及光学性能, 使石墨烯材料的开 发和研究成为继碳纳米管之后又一个国际研究热 点 ${ }^{[9 \sim 11]}$. 石墨烯是一种由碳原子以 $\mathrm{sp}^{2}$ 杂化方式连接形

\footnotetext{
* E-mail: hujh@fudan.edu.cn
}

Received April 28, 2014; revised May 7, 2014; published online May 23, 2014.

Project supported by the National Natural Science Foundation of China (Nos. 51073042, 51103026, 51373035, 51373040), the Shanghai Natural Science Funds (No. 11ZR1403100), the Shanghai Scientific and Technological Innovation Project (No. 11JC1400600), and the Shanghai Rising Star Program (No. 12QB1402900).

国家自然科学基金(Nos. 51073042，51103026，51373035，51373040)、上海市自然科学基金(No. 11ZR1403100)、上海科技创新行动计划(No. 11JC1400600)和上海市启明星计划(No. 12QB1402900)资助项目. 
成的单原子层二维晶体, 碳原子规整的排列于蜂窝状点 阵结构单元之中. 每个碳原子除了以 $\sigma$ 键与其他三个碳 原子相连之外, 剩余的 $\pi$ 电子与其他碳原子的 $\pi$ 电子形 成离域大 $\pi$ 键. 这种独特的大面积的 $\pi-\pi$ 共轭使得石墨 烯具有独一无二的热学性能、力学性能、电学性能等. 比 如, 石墨烯材料的硬度是所有已经测试材料中最大 的 $^{[12]}$, 高达 $130 \mathrm{GPa}$, 超出钢铁的一百多倍, 石墨烯的 热导率能达到 $5000 \mathrm{~W} / \mathrm{mK}$, 超出金刚石材料热导率的 2 倍 ${ }^{[13]}$, 室温下载流子在石墨烯中的迁移率可达到 $15000 \mathrm{~cm}^{2} /(\mathrm{V} \cdot \mathrm{s})$, 相当于光速的 $1 / 300^{[14]}$, 远远超过其他 半导体材料, 如锑化铟、砷化镓、硅半导体等. 同时, 石 墨烯还具有良好的加工性能和低廉的成本等优势. 众多 的优良特性, 使石墨烯在制备纳米复合材料、微电子器 件、生物传感器等方面显示出了其它材料无可比拟的优 势.

但是结构完整的石墨烯是由不含任何不稳定键的 苯六元环组合而成的二维晶体, 化学稳定性高, 其表面 呈惰性状态, 与其他介质(如溶剂等)相互作用较弱, 且 石墨烯片与片之间存在较强的范德华力, 容易产生团 聚, 使其难溶于水和常用有机溶剂, 限制了石墨烯的进 一步研究和应用. 为了充分发挥其优良性质, 并改善其 成型加工性(如提高溶解性、在基体中的分散性等), 必 须对石墨烯进行有效的功能化. 通过引入特定的官能 团, 还可以赋予石墨烯新的性质, 进一步拓展其应用领 域. 近年来, 研究人员已经做了大量的石墨烯功能化研 究来提高其分散性, 主要分为共价键功能化和非共价键 功能化两种. 非共价键功能化指通过 $\pi-\pi$ 共轭作用、离 子键以及氢键等非共价键作用使修饰分子对石墨烯进 行表面功能化, 形成稳定的分散体系. 如 Liu 等 ${ }^{[15]}$ 将带 有芘结构的聚丙烯酸 $N, N$-二甲氨基乙酯(PDMAEA)以 及负电性聚丙烯酸(PAA)用来修饰石墨烯, 制备了 $\mathrm{pH}$ 敏感的石墨烯/聚合物复合材料, 在不同的 PH值条件下, PDMAEA/石墨烯以及 PAA/石墨烯在水相和有机相中 都有明显的相转移行为. Mann 等 ${ }^{[16]}$ 将氧化石墨烯和单 链 DNA 搅拌在一起后, 水合肼还原, 制备出了 DNA 稳 定的石墨烯, 在水中可以稳定存在数月, 当不加入 DNA, 直接还原氧化石墨烯, 制备出的石墨烯材料很快 就会在水中聚集沉淀. 共价键修饰指将修饰物与石墨烯 之间通过共价键连接, 利用修饰物的空间位阻、溶解性 等来改善石墨烯的分散性和相容性等. 相对于非共价修 饰依赖的 $\pi-\pi$ 相互作用、氢键等分子间弱相互作用力易 被破坏, 共价化学键相当稳定, 不易被破坏, 因此石墨 烯的共价键修饰是目前研究较为广泛的修饰方法. 虽然 石墨烯的主体结构是由完整的苯六元环构成, 但是利用 强酸对石墨进行剥离制备氧化石墨烯的过程中会在其
边缘及缺陷部位引入很多具有活性的含氧基团, 如羒 基、羟基及环氧基等, 可以利用这些活性基团与某些官 能团的反应实现对石墨烯的共价键修饰. 如 Stankovich 等 ${ }^{[17]}$ 利用异氭酸酯与氧化石墨烯表面的羧基及羟基反 应，制备了一系列能够在 $N, N$-二甲基甲酰胺(DMF)等极 性溶剂中稳定分散的石墨烯. 相比于小分子功能化, 聚 合物功能化石墨烯更有优势, 聚合物有更多结构参数可 以调节, 如聚合物的单体种类、聚合物的拓扑结构、聚 合物的分子量、聚合物的电性等，同时聚合物修饰能赋 予石墨烯更好的机械性能包括韧性和延展性，尤其是可 以通过变换聚合物的种类和功能赋予石墨烯更多的性 能. Deng 等 ${ }^{[18]}$ 通过原子转移氮氧自由基偶合(Atom Transfer Nitroxide Radical Coupling, ATNRC)反应制备 了聚 ( $N$-异丙基丙烯酰胺) (PNIPAM)功能化的石墨烯 GO-PNIPAM，在水相及多种有机相中均表现出良好的 分散性，同时 GO-PNIPAM 对于温度刺激表现出了敏感 的相转变行为, 它的最低临界相转变温度 $\left(37{ }^{\circ} \mathrm{C}\right)$ 接近 于体温, 有前景应用于生物载药领域. 石墨烯/聚合物复 合材料不仅具有聚合物优良的机械性能、丰富的形态和 功能化, 还保持了石墨烯原来的电性能、化学性能和机 械性能等. 并且, 石墨烯的高比表面积(高达 2630 $\mathrm{m}^{2} \cdot \mathrm{g}^{-1}$, 比活性炭的比表面积高出两倍多)和生物相容性 使其可成为很好的基因/药物载体, 近年聚合物功能化

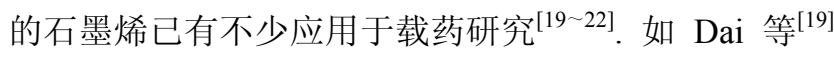
用支链聚乙烯醇接枝纳米级的氧化石墨烯片层, 并且展 现出石墨烯基材料在运输芳香族, 水溶性药物中的独特 性能. 因此, 石墨烯的聚合物功能化也越来越受到关注 和重视 ${ }^{[23 \sim 29]}$.

一般来说, 利用聚合物对石墨烯共价修饰有两种基 本的策略 ${ }^{[30]}$ : 向石墨烯接枝法 (grafting-onto)和从石墨烯 接枝法(grafting-from). 向石墨烯接枝法(grafting-onto)是 首先合成末端带有活性官能团的高分子链, 然后通过偶 联反应直接接枝到氧化石墨烯上. 2008 年, Dai 等 ${ }^{[31]}$ 利用 酰卤的酯化反应首先制备了具有生物相容性的聚乙二 醇(PEG)功能化的石墨烯, 使石墨烯具有很好的水溶性, 并且能够在血浆等生理环境下保持稳定分散; 然后再利 用 $\pi-\pi$ 相互作用首次成功地将抗肿瘤药物喜树碱衍生物 (SN38)负载到石墨烯上，开启了石墨烯在生物医药方面 的应用研究. 与小分子反应相比, 处于聚合物链上的官 能团, 由于其相对含量、官能团间的相对反应活性以及 聚合物链的屏蔽作用, 使它们所参与的化学反应受到很 大的影响, 效率较低. 而近十年, 点击化学(click chemistry $)^{[32]}$ 的发展为聚合物材料的合成提供了许多高效的 偶联反应，这种高效的 click 反应也被用于改性石墨 烯 ${ }^{[33 \sim 35]}$. 从石墨烯接枝法 (grafting-from), 是将石墨烯 
先和聚合物单体混合均匀, 然后再从石墨烯或氧化石墨 烯的片层上原位引发聚合反应，由于聚合物是从石墨烯 表面上 “长” 出来的, 可以通过改变投料比或者反应时 间来改变石墨烯表面上接枝的聚合物量. 2009 年, Ye 等 [36]就首次采用了原位自由基共聚的方法制备了两亲性 聚合物功能化的石墨烯. 但是这种传统的聚合对所接枝 的聚合物结构的控制性较差. 近年来, 活性可控聚合的 大发展也为规整聚合物功能化石墨烯提供了契机. 在已 有的几种活性可控聚合中, 包括氮氧自由基调控聚合 (nitroxide mediated polymerization, NMP) ${ }^{[37]}$ 、原子转移自 由基聚合 (atom transfer radical polymerization,

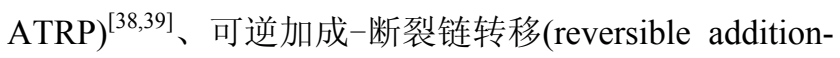
fragmentation transfer, RAFT) 聚合 ${ }^{[40,41]}$ 和单电子转移活 性自由基聚合(single-electron transfer living radical polymerization, SET-LRP) ${ }^{[42,43]}$. 其中, 单电子转移活性自 由基聚合(SET-LRP)技术可以在室温下以超快的聚合速 率制备结构可控的聚合物. 因此, 作为一种在低温下高 速进行的，并能有效控制大分子链生长的可控/ “活性” 自由基聚合技术, SET-LRP 可以与 “grafting-from” 策略 结合, 相对高效且温和地实现聚合物链在石墨烯材料上 的可控功能化改性.

本文中, 我们以天然石墨为起始原料, 采用改进的 Hummer 方法制备氧化石墨烯, 然后通过肼还原、重氮 化和酯化反应制备了表面含有 SET-LRP 引发基团的石 墨烯(G-Cl), 最后在石墨烯表面原位引发单体 $N, N$-二甲 基丙烯酰胺(DMAA)聚合, 制备 G-PDMAA 复合材料 (Scheme 1). 聚( $N, N$-二甲基丙烯酰胺) (PDMAA)作为高 性能聚合物, 同时具有极强的抗水解能力、良好的生物 相容性、优异的粘接性能等，可应用于油田化学、染料、 药物可控释放、人造器官等相关领域 ${ }^{[44 ~ 46]}$. 得到的 G-PDMAA 具有很好的溶解性和分散性, 可望用于聚合 物改性和生物材料.

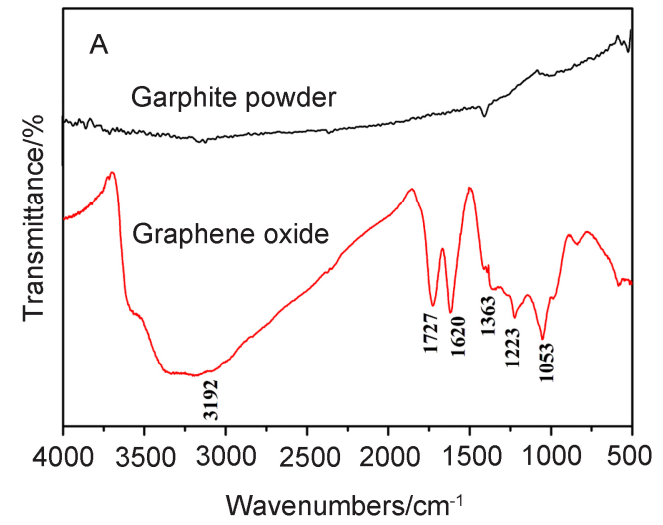

\section{1 结果与讨论}

\section{$1.1 \mathrm{G}-\mathrm{Cl}$ 引发剂的合成}

首先是氧化石墨烯的制备, 根据改进后的 Hummer 法, 利用浓硫酸以及高镇酸钾的强氧化性以及硫酸的插 层反应，分别经历低温、中温、高温反应，经过剧烈的 氧化及水解后，石墨烯片层上产生各种含氧基团，这些 含氧基团的空间位阻、静电排斥增大了石墨烯片层间距, 同时，含氧基团的亲水性也使石墨烯片层在水中有良好 的分散性. 因此，氧化石墨在去离子水中简单超声后， 可以得到淡黄色到棕褐色的氧化石墨烯水溶液, 并且这 种水溶液可以稳定存在数周而不聚集产生沉淀. 在氧化 石墨烯的红外光谱(图 1A)上, 与天然石墨相比, 可以看 到有很多官能团 $\left(3912 \mathrm{~cm}^{-1}: \mathrm{COOH}, \mathrm{OH} ; 1727 \mathrm{~cm}^{-1}\right.$ : $\mathrm{C}=\mathrm{O} ; 1223 \mathrm{~cm}^{-1}: \mathrm{C}-\mathrm{O}-\mathrm{C} ; 1363 \mathrm{~cm}^{-1}: \mathrm{C}-\mathrm{O}-\mathrm{H}$ 等)的 特征吸收峰, $1620 \mathrm{~cm}^{-1}$ 出现的峰通常被认为是层间吸 附的水分子和未被氧化的片层上的碳骨架振动峰. 通过 $\mathrm{X}$ 射线衍射(XRD), 我们对比了天然石墨粉与氧化石墨 烯的堆积结构, 在其 XRD 图(图 1B)中, 石墨与氧化石 墨出现了不同的衍射峰值, 由 Bragg 公式 $(n \lambda=2 d \sin \theta$, 其中, $n$ 为衍射级数; $\lambda$ 为 $\mathrm{X}$ 射线的波长; $d$ 为晶面间距; $\theta$ 为掠射角)可以计算出镜面间距. 天然石墨只有一个十 分尖锐的强峰，说明天然石墨的结晶度很高，其对应的 层间距为 $3.34 \AA$. 而氧化石墨烯有一个相对较宽的衍射 峰, 但其衍射角 $2 \theta$ 为 $11.4^{\circ}$, 对应的层间距为 $7.71 \AA$, 很 明显, 这比石墨层间距大一倍左右. 造成这种结果有两 方面原因; 一方面天然石墨被氧化后，石墨烯表面产生 的氧原子与碳原子在垂直于石墨烯平面的方向上以共 价键连接，从而使氧化石墨烯层间距变大，另一方面就 是含氧基团之间的静电排斥, 撑开了石墨片层.

比较石墨和氧化石墨烯的 Raman 光谱图(图 2)可以 看到, 单纯的石墨在拉曼光谱中有两个强度差别很大的

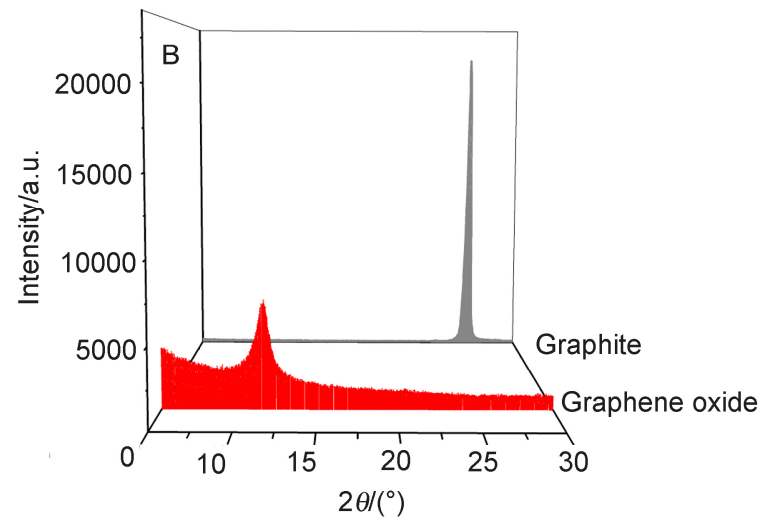

图 1 石墨和氧化石墨烯的红外谱图和 XRD 图像

Figure 1 FT-IR spectra and XRD images of graphite and grapheme oxide 

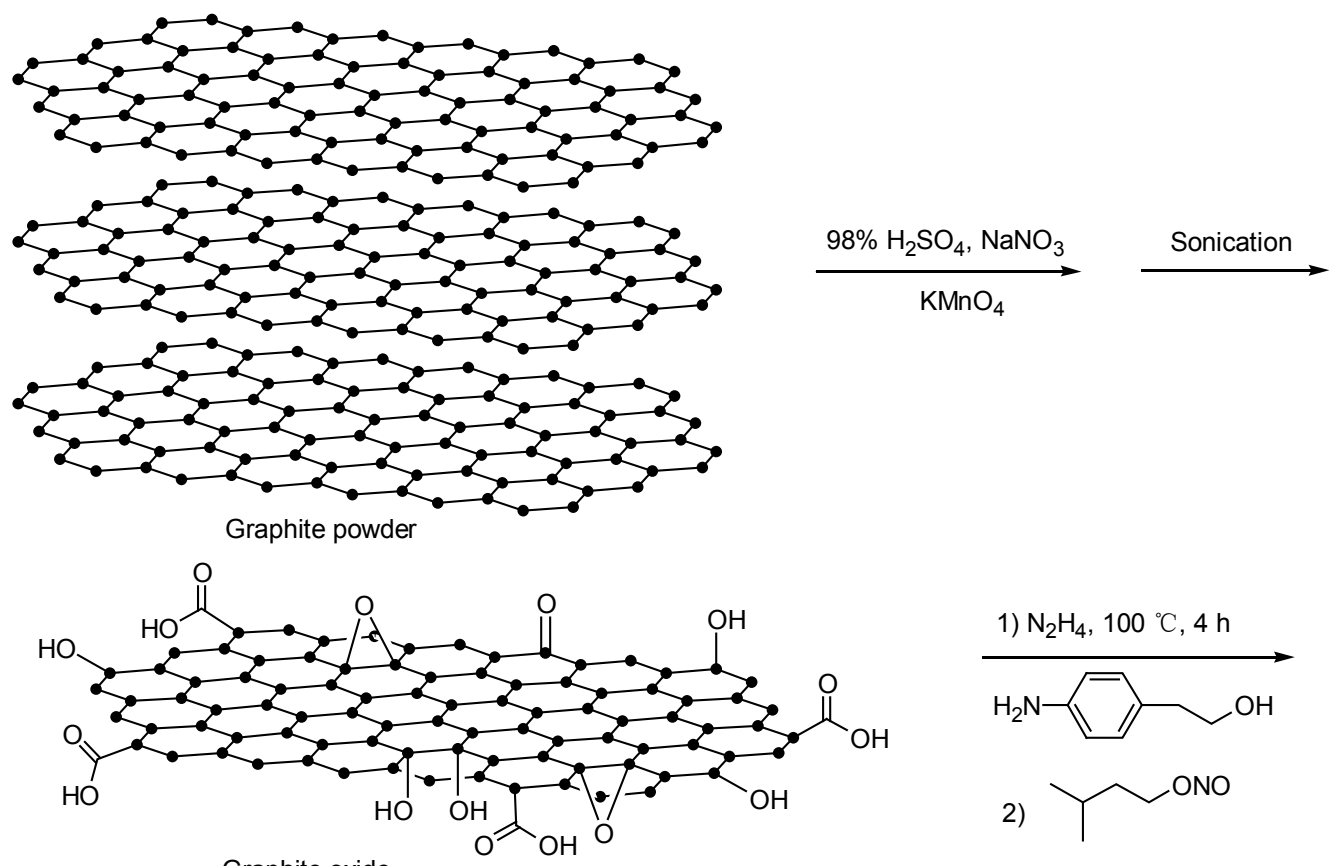

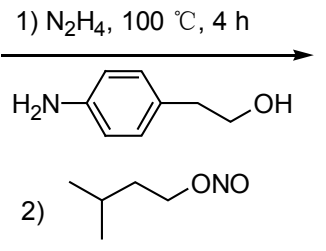

$80{ }^{\circ} \mathrm{C}$, overnight

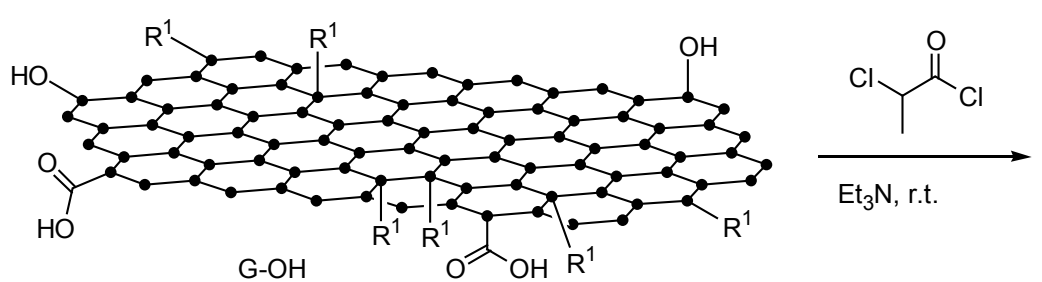

$$
\mathrm{R}^{1}=3-2 \mathrm{OH}
$$
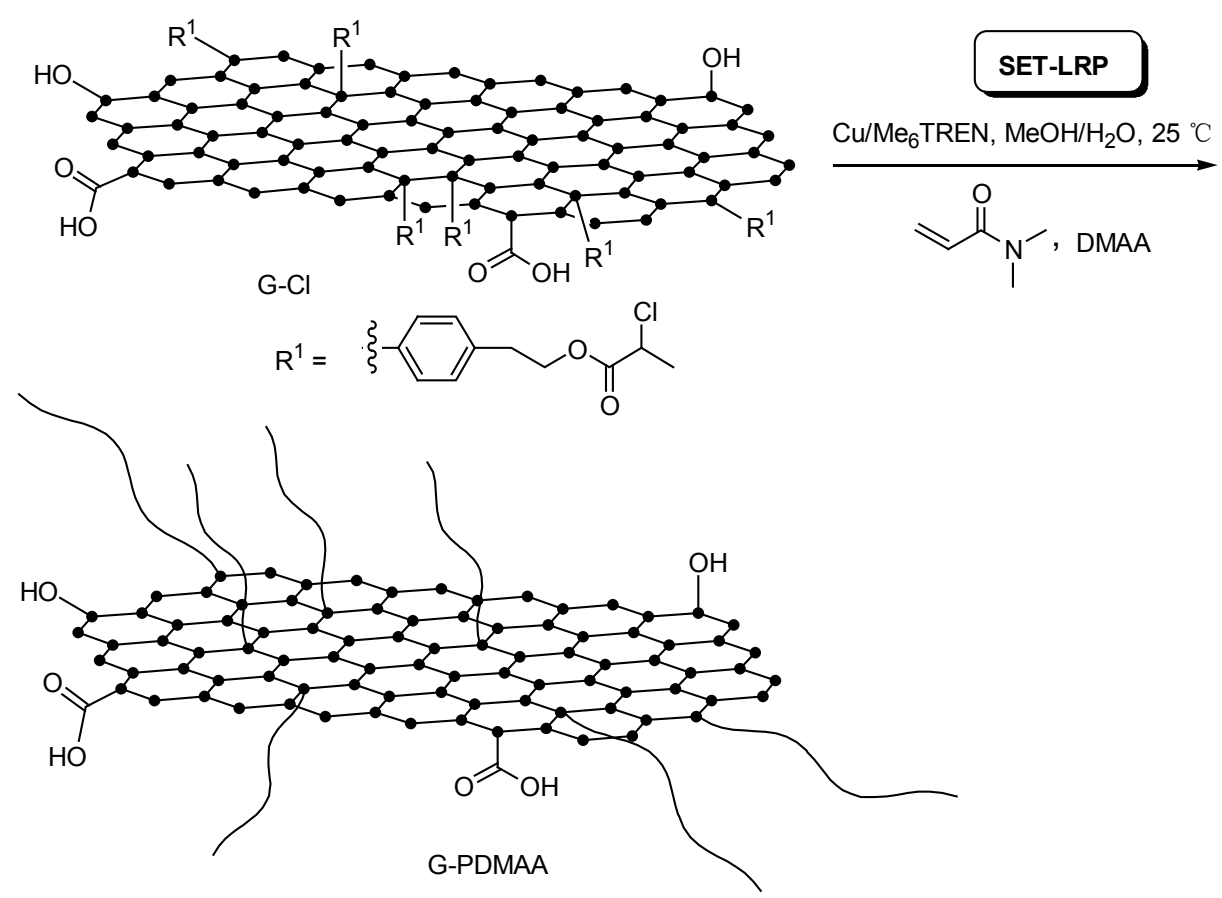

Scheme 1

峰: $1569 \mathrm{~cm}^{-1}$ 处的 $\mathrm{G}$ 峰和 $1348 \mathrm{~cm}^{-1}$ 处的 $\mathrm{D}$ 峰, $\mathrm{G}$ 峰对 应于二维的石墨晶格中 $\mathrm{sp}^{2}$ 杂化的碳原子, 它代表了有 
序的石墨烯晶格结构, 而 $\mathrm{D}$ 峰对应于石墨晶格中 $\mathrm{sp}^{3}$ 杂 化的碳原子，它代表石墨烯上的缺陷，因此从 $I_{\mathrm{D}} / I_{\mathrm{G}}$ 的比 值可以推断石墨烯表面的有序与无序程度. 天然石墨中 $\mathrm{G}$ 峰很高而 D 峰很低, 说明天然石墨中几乎所有的碳原 子都是 $\mathrm{sp}^{2}$ 杂化的. 而当石墨经过剧烈的氧化后, $\mathrm{D}$ 峰明 显增强(强度略低于 $\mathrm{G}$ 峰), 说明石墨经过剧烈的氧化后, 石墨烯结构存在一定的缺陷, 进一步验证了氧化石墨中 带有许多官能团的结论.

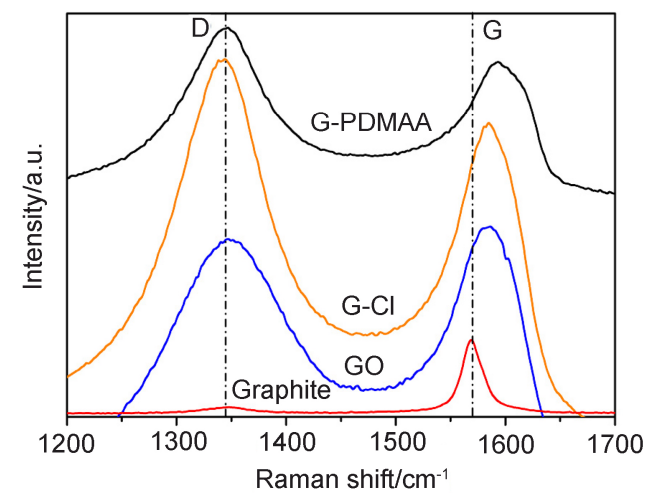

图 2 石墨、氧化石墨烯、G-Cl 和 G-PDMAA 的拉曼光谱

Figure 2 Raman spectra of graphite, GO, G-Cl, and G-PDMAA

我们进一步用原子力显微镜(Atomic Force Microscope, AFM)和透射电镜(Transmission Electron Microscope, TEM)来观察氧化石墨烯的微观形貌, 以直观的 观察并证明氧化石墨在溶剂中经过超声后确实剥离成 了单层或少层的氧化石墨烯. 我们采用滴涂(drop- casting)的方式, 吸取 $0.01 \mathrm{mg} / \mathrm{mL}$ 的氧化石墨烯水溶液, 滴 于新制云母片上, 待自然挥发干水分后, 进行 AFM 测 试. 从氧化石墨烯的 AFM 图(图 3A)中可以看出, 氧化 石墨烯表面是非常平整均一的, 以单层或者少量几层堆 叠的形式存在, 并且其高度大约为 $0.9 \mathrm{~nm}$ 左右, 这比石 墨的 $0.34 \mathrm{~nm}$ 要高很多. 我们将 $0.01 \mathrm{mg} / \mathrm{mL}$ 的氧化石墨 烯水溶液滴加到铜网上, 用 TEM 观察(图 3C), 可以看出 石墨烯的表面是均一的, 氧化石墨烯表面有暗色的条 带, 一般认为这是由于石墨烯褶皱和边缘卷起引起的.

接着, 对氧化石墨烯进行了肼还原, 同时进行重氮 化反应在石墨烯表面引入一定量的羟基, 随后与 2 -氯丙 酰氯进行酯化反应得到含 SET-LRP 引发基团的石墨烯 (G-Cl). G-Cl 的结构通过 FT-IR, Raman, HPIC 得到确认. 从 FT-IR 图(图 4A)上可以看出, $1740 \mathrm{~cm}^{-1}$ 处的峰对应于 酯羰基的振动峰, 在 $2830 \sim 2950 \mathrm{~cm}^{-1}$ 左右可以看到甲 基和亚甲基的振动峰, 说明 SET-LRP 基团 $\left[\mathrm{CO}\left(\mathrm{CH}_{3}\right) \mathrm{Cl}\right]$ 被引入到石墨烯表面. 我们看到在 $3450 \mathrm{~cm}^{-1}$ 处的羟基 以使石墨烯片层之间存在静电排斥作用从而阻止其相 互之间的团聚, 使 $\mathrm{G}-\mathrm{Cl}$ 在溶剂中有较好的分散性, 便于

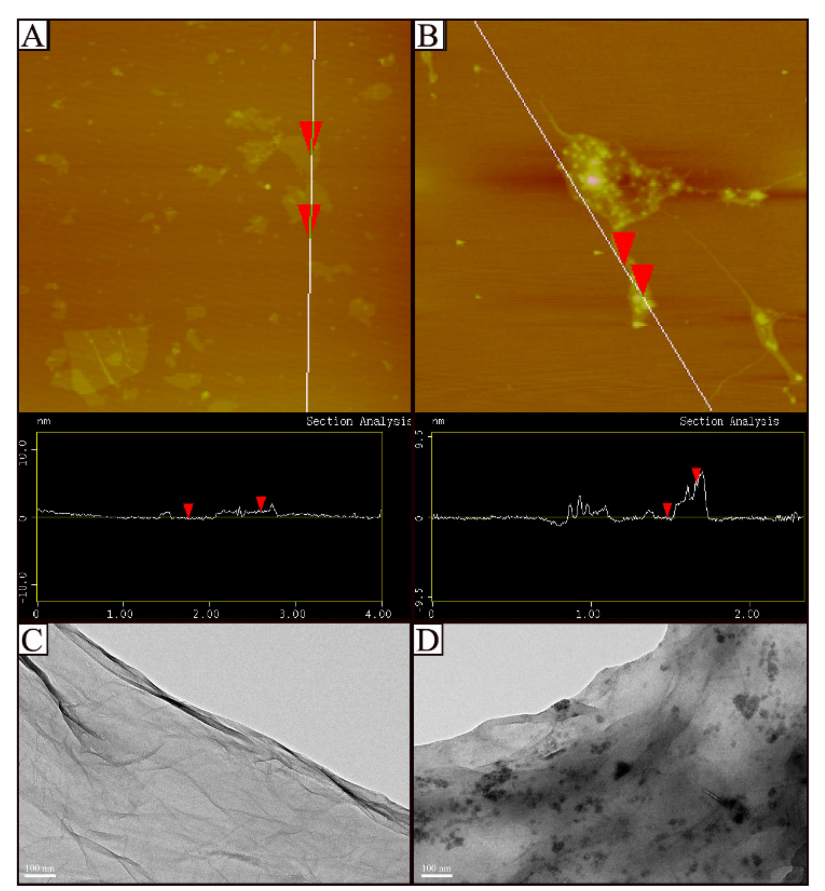

图 3 氧化石墨烯(A, C)和 G-PDMAA (B, D)的 AFM 和 TEM 图像

Figure 3 AFM and TEM images of GO (A, C) and G-PDMAA (B, D)

下一步的聚合反应.

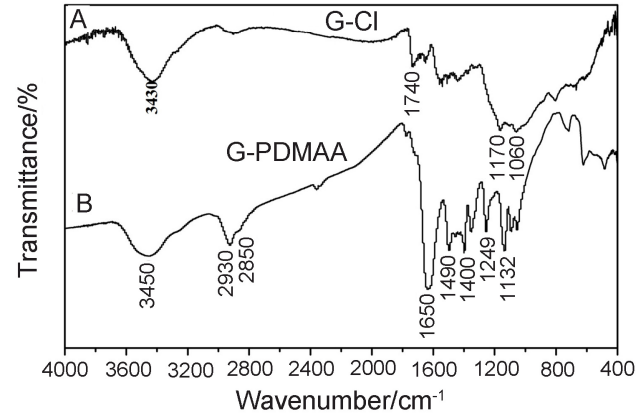

图 4 G-Cl 和 G-PDMAA 的红外谱图

Figure 4 FT-IR spectra of G-Cl and G-PDMAA

从 Raman 图(图 2)上可以看出, 其 $I_{\mathrm{D}} / I_{\mathrm{G}}(1.28)$ 比氧化 石墨烯的 $I_{\mathrm{D}} / I_{\mathrm{G}}(0.93)$ 显著有了增加, 说明 $\mathrm{G}-\mathrm{Cl}$ 中 $\mathrm{sp}^{3}$ 杂 化碳原子的增多. 理论上氧化石墨烯还原得到石墨烯后 其 $I_{\mathrm{D}} / I_{\mathrm{G}}$ 会降低, 因为 $\mathrm{D}$ 峰对应的 $\mathrm{sp}^{3}$ 杂化的碳原子减少, 但是 G-Cl 中 $\mathrm{sp}^{3}$ 杂化碳原子反而增多, 说明一定量的官 能团(SET-LRP 基团, 苯乙基等)被接枝到石墨烯表面. 为了确定石墨烯上引发剂的量, 我们采用 HPIC 对 G-Cl 上的氯含量进行测定, $\mathrm{G}-\mathrm{Cl}$ 中氯含量达到 $8.36 \mathrm{wt} \%$, 而 氧化石墨烯中不含有任何氯元素的, 这进一步确认了石 墨烯中 SET-LRP 基团 $\left[\mathrm{CO}\left(\mathrm{CH}_{3}\right) \mathrm{Cl}\right]$ 的成功接入. 根据氯 含量我们可以算出大约每一百个碳原子存在三个氯原 子引发基团, 这种接枝密度已经能够很好的引发 
DMAA 单体的聚合.

\subsection{SET-LRP 原位引发 DMAA 聚合制备石墨烯/ PDMAA 纳米复合材料(G-PDMAA)}

接着, 选用甲醇/水的混合溶剂作为聚合溶剂, 以铜 线为金属催化剂, $\mathrm{Me}_{6}$ TREN 为配体, 以含 SET-LRP 的 石墨烯 $(\mathrm{G}-\mathrm{Cl})$ 为引发剂, 从石墨烯表面原位引发单体 DMAA 进行 SET-LRP 聚合, 制得表面接枝 PDMAA 的 石墨烯复合材料. 为了更好的调控聚合物的分子量和分 子量分布, 在反应体系中加入一定量的游离引发剂(2氯丙酸甲酯), 通过外加引发剂, 一方面可以产生足够的 二价铜, 确保自由基活性中心与二价铜络合物形成休眠 种，从而实现表面 SET-LRP 可控; 另一方面，可以通过 表征游离的PDMAA 来间接验证石墨烯表面接枝聚合物 的可控性.

G-PDMAA 的结构通过 FT-IR, Raman, ${ }^{1} \mathrm{H}$ NMR, 元 素分析和 TGA 进行了表征. 在其红外光谱图(图 4B)中, 与 $\mathrm{G}-\mathrm{Cl}$ 相比, 在 $1650 \mathrm{~cm}^{-1}$ 处出现很强的峰, 对应于酰 胺中羰基的特征吸收峰, 在 2930 和 $2850 \mathrm{~cm}^{-1}$ 处的吸收 峰也明显变强, 对应于 PDMAA 亚甲基和甲基的吸收峰, 甲基由于与氮相连原 $2870 \mathrm{~cm}^{-1}$ 的吸收向低波数移动, 说明了 PDMAA 被成功接枝到石墨烯表面. 从其拉曼光 谱(图 3B)中可以看到, G-PDMAA 中存在 D 峰和 $\mathrm{G}$ 峰, 其 $I_{\mathrm{D}} / I_{\mathrm{G}}$ 的数值为 1.09 , 相比于 $\mathrm{G}-\mathrm{Cl}$ 的 $I_{\mathrm{D}} / I_{\mathrm{G}}(1.28)$ 要低, 这一现象可能的原因是: PDMAA 聚合物链的引入减少 了石墨烯表面的无序程度. 另外，与石墨相比，GO、 G-Cl 和 G-PDMAA 的 G 峰都有 $15 \sim 20 \mathrm{~cm}^{-1}$ 的蓝移, 这 可能是由于石墨烯氧化以及还原过程中形成的缺陷以 及一些孤立的双键造成的 ${ }^{[47]}$.

表 $1 \mathrm{G}-\mathrm{Cl}$ 与 G-PDMAA 的元素分析结果

Table 1 Elementary analysis of G-Cl 与 G-PDMAA

\begin{tabular}{lcccc}
\hline Sample & $\mathrm{C}$ & $\mathrm{H}$ & $\mathrm{N}$ & $\mathrm{C} / \mathrm{H}$ molar ratio \\
\hline G-Cl & 70.06 & 3.43 & 3.80 & 1.70 \\
G-PDMAA & 58.68 & 8.66 & 12.00 & 0.56 \\
\hline
\end{tabular}

通过元素分析, 我们对比了聚合物接入前后石墨烯 样品的碳、氢、氮含量, 从表 1 中可以看到, 由于含氮 聚合物的引入, 氮含量得到明显提高. $\mathrm{G}-\mathrm{Cl}$ 的 $\mathrm{C} / \mathrm{H}$ 物质 的量比为 1.70 , 而 G-PDMAA 的 $\mathrm{C} / \mathrm{H}$ 物质的量比为 0.56 , $\mathrm{C} / \mathrm{H}$ 值的降低表明 G-PDMAA 中 $\mathrm{H}$ 含量大大增加, 进一 步说明石墨烯中 PDMAA 的引入. 从氮含量的差值 8.20 $\mathrm{wt}^{2} \%(\mathrm{NG}-\mathrm{PDMAA}-\mathrm{NG}-\mathrm{Cl}=12.00 \%-3.80 \%=8.20 \%)$, 可以推算出石墨烯表面上接枝的聚合物含量为: $58 \mathrm{wt} \%$. 在 G-PDMAA 的氢谱(图 5)中, 可以看到 PDMAA 的特 征峰, $\delta 1.62$ 处峰对应于 PDMAA 主链 $\mathrm{CHCH}_{2}$ 上的氢, $\delta$ 2.85 处的峰对应于 PDMAA 链上的 $\mathrm{N}\left(\mathrm{CH}_{3}\right)_{2}$ 的氢, 表明
PDMAA 被成功接枝到石墨烯表面. 从聚合体系中游离 聚合物的 GPC 表征中, 可以看到游离的 PDMAA 分子量 分布较窄 $\left(M_{\mathrm{w}} / M_{\mathrm{n}}=1.28\right)$, 说明整个聚合体系是可控的, 因此从石墨烯表面引发的聚合也是活性可控的, 即表面 接枝的 PDMAA 是规整的.

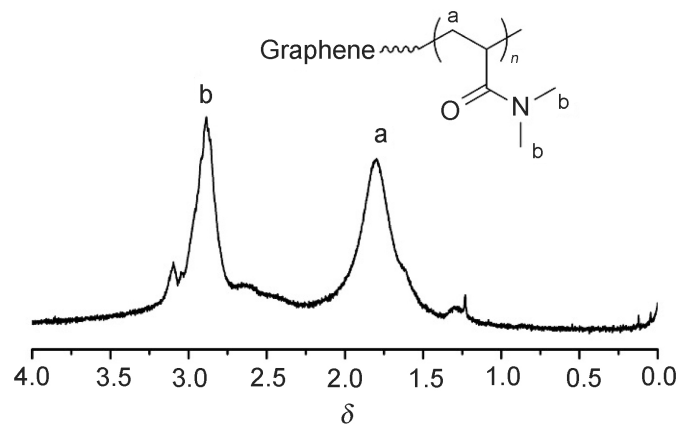

图 5 G-PDMAA 在気代氯仿中的氢谱

Figure $5{ }^{1} \mathrm{H}$ NMR spectra of G-PDMAA in $\mathrm{CDCl}_{3}$

石墨烯上所接枝聚合物的量也可以通过 TGA 来检 测. 我们知道，当氧化石墨受热时，其表面上的含氧基 团很容易分解离去, 从而导致其耐热性很差. 从 $\mathrm{G}-\mathrm{Cl}$ 和 G-PDMAA 的 TGA 曲线(图 6)中可以发现, $\mathrm{G}-\mathrm{Cl}$ 热稳定 性有显著的提高，当温度升到 $700{ }^{\circ} \mathrm{C}$ 时, $\mathrm{G}-\mathrm{Cl}$ 的残余量 为 $55.78 \mathrm{wt} \%$, 而 G-PDMAA 在 300 和 $450{ }^{\circ} \mathrm{C}$ 之间有一 个明显的失重, 这可归因于聚合物 PDMAA 在此温度区 间受热分解, G-PDMAA 在 $700{ }^{\circ} \mathrm{C}$ 的残余质量分数为 $11.46 \mathrm{wt} \%$. 假如在此温度区间所有的热失重都是由于 接枝的聚合物引起的，那么石墨烯上接枝聚合物的量为 $44.32 \mathrm{wt} \%(=55.78 \% \sim 11.46 \%)$. 这个数值与从元素分 析上通过氮含量的差值得到的接枝聚合物的量 $58 \mathrm{wt} \%$ 有点差距，这可能是由于不同的测试方法造成.

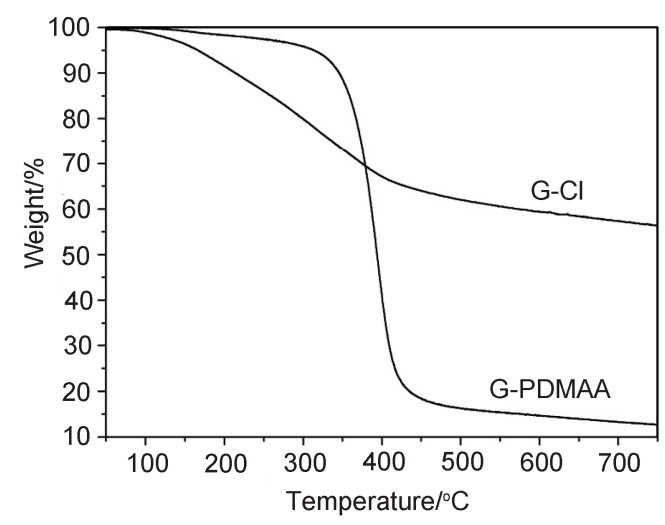

图 $6 \mathrm{G}-\mathrm{Cl}$ 和 G-PDMAA 的 TGA 曲线

Figure 6 TGA curves of G-Cl and G-PDMAA

同样地, 我们通过 AFM 和 TEM 直观地观察 G-PDMAA 的表面形貌. 在 G-PDMAA 的 AFM 图(图 3B) 中, 与氧化石墨烯相比, G-PDMAA 表面比较粗粘并且 
可以看到一些突出的突起部分, 这主要是石墨烯表面聚 合物的包裹以及褶皱引起的. G-PDMAA 的厚度从氧化 石墨烯的 $0.934 \mathrm{~nm}$ 显著增加到 $4.190 \mathrm{~nm}$, 说明石墨烯表 面上聚合物的存在. 在 G-PDMAA 的 TEM 图(图 3D)中, 与氧化石墨烯带有少部分褶皱的表面相比, G-PDMAA 上有许多突起, 这些突起就是聚合物相互之间褶皱以及 包裹引起的, 这也直观的证明了石墨烯表面接枝上了 PDMAA 聚合物.

最后, 我们研究了 G-PDMAA 在不同溶剂中的分散 性. 仅通过简单的超声后, G-PDMAA 可以很好的分散 在多种有机溶剂和水中(图 7). G-PDMAA 良好的分散性 应当大部分归因于聚合物的成功引入, 因为按照文献报 道, 还原后的石墨烯在不依赖表面活性剂的情况下, 是 无法分散于非极性溶剂中的 ${ }^{[48 \sim 50]}$. 这归功于石墨烯表 面上的聚合物之间存在静电排斥、位阻效应和聚合物本 身的溶解性, G-PDMAA 在有机溶剂中的良好分散性为 石墨烯材料的进一步应用提供了非常重要的平台.

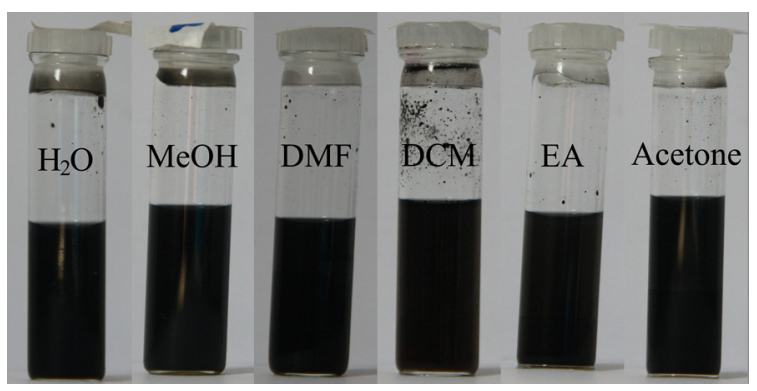

图 7 G-PDMAA 在有机溶剂和水中分散的数码图像

Figure 7 Digital photos of G-PDMAA dispersed in different organic solvents and aqueous solvent

\section{2 结论}

用改进的 Hummers 法制备了氧化石墨，通过对氧 化石墨烯还原过程中进行重氮化反应和酯化反应在石 墨烯表面引入可以引发 SET-LRP 聚合的引发基团, 然 后在室温条件下以 $\mathrm{Cu} / \mathrm{Me}_{6} \mathrm{TREN}$ 为催化体系在甲醇和 水的混合溶剂中从石墨烯表面上进行原位 SET-LRP 聚 合制备了 G-PDMAA 纳米复合材料, G-PDMAA 在常规 的溶剂中有良好的分散性和溶解性.

\section{3 实验部分}

\section{1 仪器与试剂}

核磁共振 $\left({ }^{1} \mathrm{H}\right.$ NMR): Bruker AM-300, 溶剂为気代 氯仿，四甲基硅(TMS)为内标. 红外光谱(FT-IR): Nicolet AVATAR-360, 所有红外谱图累加 32 次, 分辨率为 4.0 $\mathrm{cm}^{-1}$. 元素分析(E.A.): Elemantar VARIOEL, Heraeus 1106, Carlo-Erba 1108. 离子色谱(HPIC): 样品经氧瓶燃 烧法处理, 仪器 Dionex DX-500, 分离柱: IonPac
$\mathrm{AG}_{14}-\mathrm{AS}_{14}$, 检测方式: 电导, 淋洗液: $\mathrm{NaHCO}_{3} 0.00010$ $\mathrm{mol} / \mathrm{L}+\mathrm{Na}_{2} \mathrm{CO}_{3} 0.0035 \mathrm{~mol} / \mathrm{L}$. 拉曼光谱(RAMAN): Jobin Yvon LabRAM HR 800 UV 共聚焦拉曼显微镜，激发 波长为 $514.5 \mathrm{~nm}$, 粉末样品经干燥除溶剂后室温下直接 平铺在载玻片上进行观察. 凝胶渗透色谱(GPC): Waters 1515 凝胶色谱仪(HR3 (500～30000), HR4 (5000 600000), HR5 (50000 4000000), $7.8 \mathrm{~mm} \times 300 \mathrm{~mm}$ ), 采 用 Waters 2410 示差折光检测器. 用 THF 将聚合物配成 $1 \mathrm{mg} / \mathrm{mL}$ 的溶液, 淋洗液为 $\mathrm{THF}$, 流速为 $1 \mathrm{~mL} / \mathrm{min}$, 采 用线性聚苯乙烯标样对分子量进行校正. 透射电子显微 镜(TEM): Philips CM 120 型透射电子显微镜(加速电压 $80 \mathrm{kV}$ ). 原子力显微镜(AFM): 在 Nanoscope IVa-MultiMode 型 AFM 上进行测试, 采用 Veeco PTESP7 型探针, 在室温空气中采用 Tapping Mode 模式进行观察, 样品 超声分散于溶剂中，然后旋涂在云母片上. $X$ 射线衍射 仪(XRD): 样品晶体结构利用 Philips X'Pert PRO 型 X 射 线衍射仪测量, 其中 $\mathrm{CuK} \alpha(\lambda=1.541 \AA)$, 测试电压和电 流分别为 $40 \mathrm{kV}$ 和 $40 \mathrm{~mA}$, 扫描速率 $2 \theta=0.0334\left(^{\circ}\right) / \mathrm{s}$, 扫 描角度范围是从 $25^{\circ}$ 到 $90^{\circ}$. 热失重分析(TGA): 样品热 稳定性在 TA Q500 型热失重分析仪上得到，一般扫描范 围为 $50{ }^{\circ} \mathrm{C}$ 升温到 $800{ }^{\circ} \mathrm{C}$, 升温速率为 $10{ }^{\circ} \mathrm{C} / \mathrm{min}$, 氮气 流速为 $50 \mathrm{~mL} / \mathrm{min}$. 测试前, 粉末样品需研磨确保传热 均匀. 超声处理: 采用 DS-3510 型水浴超声仪进行超声, 频率为 $40 \mathrm{kHz}$.

$\mathrm{N}, \mathrm{N}$-二甲基甲酰胺(DMF) 先经硫酸镁干燥数天, 减 压蒸馏备用. $N, N$-二甲基丙烯酰胺(Aldrich, 97\%)过中性 氧化铝柱除去阻聚剂, 密封保存备用. 反应所用水均为 去离子水. 电镜测试所用水为超纯水. 三乙胺 $\left(\mathrm{Et}_{3} \mathrm{~N}\right)$ 先 经 $\mathrm{KOH}$ 干燥数天, 在 $\mathrm{CaH}_{2}$ 存在下回流蒸出, 现蒸现用. 水合肼(上海大合化学品有限公司), 双氧水(国药), 硝酸 钠(国药), 高镇酸钾(国药)及其它国产试剂未注明的, 均 未经处理直接使用. 石墨粉 (graphite powder, Aldrich, $<20 \mu \mathrm{m}$, synthetic $=$, 2-(4-氨基笨)乙醇(TCI, 98\%), 铜线 (Alfa Aesar, 99.99\%), 亚硝酸异戊酯(TCI, 98\%)，2-氯丙 酸甲酯(阿拉丁, 98\%)，2-氯丙酰氯(阿拉丁，97\%)未经纯 化直接使用. 三(2-二甲氨基乙基)胺 [Tris(2-(dimethylamine)ethyl)amine, $\mathrm{Me}_{6}{ }_{\mathrm{TREN}}$ 按照文献合成 ${ }^{[51]}$.

\section{2 实验方法}

\section{2 .1 氧化石墨的制备}

依次将 $5 \mathrm{~g}$ 石墨, $5 \mathrm{~g}$ 硝酸钠, $230 \mathrm{~mL} 98 \%$ 浓硫酸加 入到干燥的 $1 \mathrm{~L}$ 烧杯中, 然后置于 $0{ }^{\circ} \mathrm{C}$ 冰水浴中, 缓慢 加入 $15 \mathrm{~g}$ 高镇酸钾 (注: 维持体系温度不要超过 $10{ }^{\circ} \mathrm{C}$ ), 加完后搅拌 $30 \mathrm{~min}$ 进行低温反应，随后体系升温至 $35 \sim 40{ }^{\circ} \mathrm{C}$, 搅拌 $4 \mathrm{~h}$ 后加入 $230 \mathrm{~mL}$ 去离子水水解, 搅 拌 $15 \mathrm{~min}$ 后, 加入 $700 \mathrm{~mL}$ 去离子水淬灭反应, 然后加 
入双氧水摚拌一段时间后, 加入 $5 \%$ 稀盐酸洗涤, 过滤, 离心，直至滤液呈中性，最后真空干燥.

\subsubsection{G-OH 的合成}

重氮化反应: $500 \mathrm{mg}$ 氧化石墨首先超声分散于 250 $\mathrm{mL}$ 去离子水中, 然后将反应瓶置于 $100{ }^{\circ} \mathrm{C}$ 油浴中, 保 持回流的状态下，缓慢加入 $2.5 \mathrm{~mL} 80 \%$ 的水合肼，加完 后继续搅拌 $4 \mathrm{~h}$, 氮气保护下降温至 $80{ }^{\circ} \mathrm{C}$, 然后加入 $4 \mathrm{~g}$ 2-(4-氨基苯)乙醇和 $3 \mathrm{~mL}$ 亚硝酸异戊酯, 搅拌过夜. 冷 至室温后, 用 $0.22 \mu \mathrm{m}$ 的聚四氟乙烯膜过滤, 得到的黑 色滤渣用大量的四氢呋喃、丙酮、去离子水和 DMF 洗 涤, 然后将得到的产物超声分散于 DMF 中, 重复洗涤 三遍，得到的黑色固体产物 G-OH 真空干燥至恒重.

\subsubsection{G-Cl 的合成}

氮气保护下将 $\mathrm{G}-\mathrm{OH}(140 \mathrm{mg})$ 、三乙胺 $(2.3 \mathrm{~mL}, 16$ $\mathrm{mmol}$ )和干燥的 DMF $(40 \mathrm{~mL})$ 加入到 $100 \mathrm{~mL}$ 的三口烧 瓶中 (使用前真空火烤三次). 然后将体系置于 $0{ }^{\circ} \mathrm{C}$ 冰水 浴中, 搅拌 $10 \mathrm{~min}$ 后, 缓慢滴加 2-氯丙酰氯 $(1.6 \mathrm{~mL}, 16$ mmol)的 DMF $(10 \mathrm{~mL})$ 溶液, 继续在 $0{ }^{\circ} \mathrm{C}$ 搅拌 $1 \mathrm{~h}$, 然后 回至室温摚拌过夜. 溶液用 $0.22 \mu \mathrm{m}$ 的滤膜过滤, 用大 量的 DMF、去离子水和四氢呋喃洗涤, 在 $40{ }^{\circ} \mathrm{C}$ 真空干 燥箱中干燥过夜.

\subsection{4 通过 SET-LRP 原位制备石, 墨烯/PDMAA 纳米 复合材料 G-PDMAA}

氮气的保护下, $\mathrm{G}-\mathrm{Cl}(20 \mathrm{mg})$ 加入到 $25 \mathrm{~mL}$ 的 Schlenk 瓶中(使用前真空火烤三次), 抽换氮气三次后, 加入 $\mathrm{MeOH} / \mathrm{H}_{2} \mathrm{O}(1.8 \mathrm{~mL} / 0.2 \mathrm{~mL})$, 将体系超声 $3 \mathrm{~h}$, 然后 依次加入 2-氯丙酸甲酯(5.5 uL, $0.0485 \mathrm{mmol}), N, N$-二甲 基丙烯酰胺 $(2 \mathrm{~mL}, 19.42 \mathrm{mmol}$ )和铜线(长度: $10 \mathrm{~cm}$, 直 径: $0.64 \mathrm{~mm}$, 包裹在搅拌子上), 冻抽-解冻三次, 室温 搅拌 $10 \mathrm{~min}$ 后加入 $\mathrm{Me}_{6} \mathrm{TREN}$ (3.2 $\left.\mu \mathrm{L}, 0.0106 \mathrm{mmol}\right)$, 室 温反应 $6 \mathrm{~h}$ 后用液氮淬灭反应. 用过量的 THF 稀释产物, 用 $0.22 \mu \mathrm{m}$ PVDF 的滤膜过滤, 并用大量的去离子水和 DMF 洗涤滤渣, 所得黑色固体产物在 $40{ }^{\circ} \mathrm{C}$ 的真空烘箱 中干燥至恒重. 同时, 滤液过中性氧化铝柱除去铜盐, 并在正己烷中沉降三次, 紧接着, PDMAA 聚合物在真 空烘箱中干燥至恒重(GPC: DMF 作为流动相, $M_{\mathrm{n}}=$ $\left.25000 \mathrm{~g} / \mathrm{mol}, M_{\mathrm{w}} / M_{\mathrm{n}}=1.28\right)$.

\section{References}

[1] Kroto, H. W.; Heath, J. R.; O'Brien, S. C.; Curl, R. F.; Smalley, R. E. Nature 1985, 318, 162 .

[2] Iijima, S. Nature 1991, 354, 56

[3] Reed, C. A.; Kim, K. C.; Bolskar, R. D.; Mueller, L. J. Science 2000, 289, 101.

[4] Cumings, J.; Zettl, A. Science 2000, 289, 602.

[5] Vigolo, B.; Coulon, C.; Maugey, M.; Zakri, C.; Poulin, P. Science 2005, 309, 920 .
[6] Jarillo-Herrero, P.; Van-Dam, J. A.; Kouwenhoven, L. P. Nature 2006, 439, 953.

[7] Otero, G.; Biddau, G.; Sanchez-Sanchez, C.; Caillard, R.; López, M. F.; Rogero, C.; Palomares, F. J.; Cabello, N.; Basanta, M. A.; Ortega, J.; Méndez, J.; Echavarren, A. M.; Pérez, R.; Gómez-Lor, B.; Martín-Gago, J. A. Nature 2008, 454, 865.

[8] Novoselov, K. S.; Geim, A. K.; Morozov, S. V.; Jiang, D.; Zhang, Y.; Dubonos, V.; Grigorieva, I. V.; Firsov, A. A. Science 2004, 306, 666.

[9] Berger, C.; Song, Z. M.; Li, X. B.; Wu, X. S.; Brown, N.; Naud, C.; Mayou, D.; Li, T. B.; Hass, J.; Marchenkov, A. N.; Conrad, E. H.; First, P. N.; de Heer, W. A. Science 2006, 312, 1191.

[10] Meyer, J. C.; Geim, A. K.; Katsnelson, M. I.; Novoselov, K. S.; Booth, T. J.; Roth, S. Nature 2007, 446, 63.

[11] Miller, D. L.; Kubista, K. D.; Rutter, G. M.; Ruan, M.; de Heer, W. A.; First, P. N.; Stroscio, J. A. Science 2009, 324, 924.

[12] Liu, Y. L.; Xie, B.; Zhang, Z.; Zheng, Q. S.; Xu, Z. P. J. Mech. Phys. Solids. 2012, 60, 591.

[13] Balandin, A. A.; Ghosh, S.; Bao, W. Z.; Calizo, I.; Teweldebrhan, D.; Miao, F.; Lau, C. N. Nano Lett. 2008, 8, 902.

[14] Chen, J. H.; Jang. C.; Xiao, S. D.; Ishigami, M.; Fuhrer, M. S. Nat. Nanotechnol. 2008, 3, 206.

[15] Liu, J. Q.; Tao, L.; Yang, W. R.; Li, D.; Boyer, C.; Wuhrer, R.; Davis, T. P. Langmuir 2010, 26, 10068.

[16] Patil, A. J.; Vickery, J. L.; Scott, T. B.; Mann, S. Adv. Mater. 2009, $21,3159$.

[17] Stankovich, S.; Piner, R. D.; Nguyen, S. T.; Ruoff, R. S. Carbon 2006, 44, 3342.

[18] Deng, Y.; Li, Y. J.; Dai. J.; Lang, M. D.; Huang, X. Y. J. Polym. Sci. Polym. Chem. 2011, 49, 1582.

[19] Liu, Z.; Robinson, J. T.; Sun, X. M.; Dai, H. J. J. Am. Chem. Soc. 2008, 130, 10876.

[20] Mohanty, N.; Berry, V. Nano Lett. 2008, 8, 4469.

[21] Xu, Z. Y.; Li, Y. J.; Shi, P.; Wang, B. C.; Huang, X. Y. Chin. J. Org. Chem. 2013, 33, 573 (in Chinese). (徐志远, 李永军, 史萍, 王博婵, 黄晓宇, 有机化学, 2013, 33, 573.)

[22] Xu, Z. Y.; Li, Y. J.; Shi, P.; Wang, B. C. Huang, X. Y. Chin. J. Org. Chem. 2013, 33, 2162 (in Chinese).

(徐志远, 李永军, 史萍, 王博婵, 黄晓宇, 有机化学, 2013, 33, 2162.)

[23] Shen, J.; Hu, Y.; Li, C.; Qin, C.; Ye, M. Small 2009, 5, 82.

[24] Fang, M.; Wang, K. G.; Lu, H. B.; Yang, Y. L.; Nutt, S. J. Mater. Chem. 2009, 19, 7098.

[25] Huang, X.; Yin, Z.; Wu, S.; Qi, X.; He, Q.; Zhang, Q.; Yan, Q.; Boey, F.; Zhang, H. Small 2011, 7, 1876.

[26] Singh, V.; Joung, D.; Zhai, L.; Das, S.; Khondaker, S. I.; Seal, S. Prog. Mater. Sci. 2011, 56, 1178.

[27] Du, J.; Cheng, H. M. Macromol. Chem. Phys. 2012, 213, 1060.

[28] Dai, J.; Lang, M. D. Acta Chim. Sinica 2012, 70, 1237 (in Chinese). (戴静, 郎美东, 化学学报, 2012, 70, 1237.)

[29] Paulus, G. L. C.; Wang, Q. H.; Strano, M. S. Acc. Chem. Res. 2013, $46,160$.

[30] Salavagione, H. J.; Martinez, G.; Ellis, G. Macromol. Rapid Commun. 2011, 32, 1771.

[31] Liu, Z.; Robinson, J. T.; Sun, X. M.; Dai, H. J. J. Am. Chem. Soc. 2008, 130, 10876.

[32] Kolb, H. C.; Finn, M. G.; Sharpless, K. B. Angew. Chem., Int. Ed. 2001, 40, 2004.

[33] Cao, Y. W.; Lai, Z. L.; Feng, J. C.; Wu, P. Y. J. Mater. Chem. 2011, $21,9271$.

[34] Jin, Z.; McNicholas, T. P.; Shimizu, S.; Strano, M. S. Chem. Mater. 
2011, 23, 3362.

[35] Salavagione, H. J. Chem. Eur. J. 2012, 18, 4965.

[36] Shen, J. F.; Hu, Y. H.; Li, C.; Qin, C.; Ye, M. X. Small 2009, 5, 82.

[37] Hawker, C. J.; Bosman, A. W.; Harth, E. Chem. Rev. 2001, 101, 3661.

[38] Kamigaito, M.; Ando, T.; Sawamoto, M. Chem. Rev. 2001, 101, 3689 .

[39] Coessens, V.; Pintauer, T.; Matyjaszewski, K. Prog. Polym. Sci. 2001, 26, 337.

[40] Perrier, S.; Takolpuckdee, P. J. Polym. Sci., Part A: Polym. Chem. 2005, 43, 5347.

[41] Lowe, A. B.; McCormick, C. L. Prog. Polym. Sci. 2007, 32, 283.

[42] Rosen, B. M.; Percec, V. Chem. Rev. 2009, 109, 5069.

[43] Levere, M. E.; Nguyen, N. H.; Sun, H. J.; Percec, V. Polym. Chem. 2013, 4, 686 .
[44] Tomczak, S. J.; Hogen-Esch, T. E. Polym. Prepr. 2001, 42, 562.

[45] Santos, W. L. F.; Porto, M. F.; Muniz, E. C.; Povh, N. P.; Rubira, A. F. J. Supercrit. Fluids 2001, 19, 177.

[46] Bromberg, L.; Grosberg, A. Y.; Matsuo, E. S.; Suzuki, Y.; Tanaka, T. J. Chem. Phys. 1997, 106, 2906.

[47] Deng, Y.; Li, Y. J.; Dai. J.; Lang, M. D.; Huang, X. Y. J. Polym. Sci., Part A: Polym. Chem. 2011, 49, 4747.

[48] Rao, C. N. R.; Biswas, K.; Subrahmanyam, K. S.; Govindaraj, A. J. Mater. Chem. 2009, 19, 2457

[49] Boukhvalov, D. W.; Katsnelson, M. I. J. Phys.: Condens. Matter 2009, 21.

[50] Liang, Y. Y.; Wu, D. Q.; Feng, X. L.; Mullen, K. Adv. Mater. 2009, 21,1679 .

[51] Queffelec, J.; Gaynor, S. G.; Matyjaszewski, K. Macromolecules 2000, 33, 8629 .

(Cheng, F.) 